\title{
Inguinal Hernia Repair in Patients with Cirrhosis is not Associated with Increased Risk of Complications and Recurrence
}

\author{
Saboor Khan
}

Published online: 23 March 2011

(c) Société Internationale de Chirurgie 2011

John was wheeled in by his weary wife. He was unkempt, malnourished with hollow eyes, and alcohol on his breath. "How is your hernia, John?" I asked, "Oh, not too bad, but it aches and I can't always push it back." His wife rolled her eyes. "Never stopped you from walking to buy your booze!" John was 43 with end-stage alcoholic liver disease and a liking for Codeine. Like many previous Registrars, I dodged the bullet and deferred his operation. He was admitted 4 weeks later with an incarcerated hernia, small bowel obstruction, and hepatic decompensation. Should I have listed him when I had the chance? The question still haunts me.

The literature is replete with data confirming the link between poor hepatic functional reserve (as measured by Child-Pugh class and the MELD score) and increased perioperative mortality, irrespective of the type of surgery [1]. The obvious risks include hepatic decompensation, hematological abnormalities (low platelet count, coagulopathy), and renal and portal hypertension-related problems (e.g., ascites, variceal bleeding, recanalization of umbilical veins leading to perioperative bleeding and pulmonary hypertension).

The question of hernia repair in such patients has taxed surgeons, dogged by the paradox of a low-risk procedure in a high-risk population. The early data on hernia repair in this population is understandably harrowing. The crux of the matter, in my opinion, has not been adequately addressed. The current study by Lo et al. [2] reports excellent results for elective inguinal hernia operation in this group. In fact, their results for cirrhotic patients are no different from those in noncirrhotic patients. However,

Saboor Khan $(\square)$

Department of General Surgery, University Hospital Coventry and Warwickshire, Coventry, UK

e-mail: hpb.surgery@gmail.com they fail to emphasize enough the one crucial element: adequate and meticulous multidisciplinary, preoperative patient preparation. Furthermore, they do not give enough credit to their hepatology colleagues or the astute clinical team that makes the decision regarding the timing of the operation. It is this risk modification "prior" to elective surgical repair and the close postoperative supervision that I believe shifted the balance in this study.

For those of us who have hepatological oversight in the management of such patients, the onus is on us to lower the perioperative risk. The timing (determining baseline optimal liver function), correction of malnutrition, use of blood or blood products (e.g., platelets, plasma), use of medications (diuretics, $\beta$-blockers, somatostatin analogs), and interventions [transjugular intrahepatic portosystem shunt (TIPS), hepatic transplantation] can have a major impact on outcome. For the less fortunate, single-handed surgeon, the assessment of these patients will continue to be problematic.

The results of Oh et al. quash some old dogma about inguinal hernia repair in patients with ascites and ChildPugh C status [2]. Should these encouraging outcomes entice us to be bolder in the repair of symptomatic inguinal hernias in this group? The answer, in my view, is only if you can reproduce the clinical acumen and expertise of a multidisciplinary team when managing these complex patients.

\section{References}

1. Teh SH, Nagorney DM, Stevens SR et al (2007) Risk factors for mortality after surgery in patients with cirrhosis. Gastroenterology 132:1261-1269

2. Oh HK, Kim H, Ryoo S et al (2011) Inguinal hernia repair in patients with cirrhosis is not associated with increased risk of complications and recurrence. World J Surg. doi:10.1007/s00268011-1007-9 